\title{
Lead-lag Controller Design for Time Delay Systems Using Genetic Algorithms
}

\author{
Nidhal BEN HASSEN ${ }^{1}$, Karim SAADAOUI ${ }^{1,2}$, Mohamed BENREJEB ${ }^{1}$ \\ ${ }^{1}$ Université de Tunis El Manar, Ecole Nationale d'Ingénieurs de Tunis, \\ Laboratoire de Recherche en Automatique (LARA), B.P. 37, 1002 Tunis, Le Belvédère, Tunisia. \\ ${ }^{2}$ Computer Engineering Department, \\ College of computers and Information Technology, Taif University, Taif, Saudi Arabia. \\ Nidhal.Benhassen@enit.rnu.tn, karim.saadaoui@isa2m.rnu.tn, mohamed.benrejeb@enit.rnu.tn
}

Abstract: In this article, the set of all stabilizing lead-lag controllers applied to a class of linear time delay systems is obtained. The original problem is divided into two sub-problems with the help of Kharitonov's Lemma. This essential step allows the application of the D-decomposition method and the determination of the complete set of stability regions. In the second part of this paper, stability regions are used as search space for Genetic Algorithms (GA) to minimize several performance indices of the closed loop system. An example is given to show the effectiveness of the proposed controller design.

Keywords: Time delay systems; Second order phase lead-lag controller; Stability; D-decomposition; Genetic Algorithms

\section{Introduction}

It is well known that low fixed-order controllers such as P, PI, PID, first-order, phase lead and phase lag controllers are widely used in industry processes. For this reason, many researchers were interested in determining the set of all stabilizing low-order controllers for linear time invariant systems; see [25], [26] and [31] and the references therein.

Time delay inherently exists in many physical systems such as chemical, mechanical and hydraulic systems [3], [22] and [24]. Therefore, it is natural that the above line of research was extended to time delay systems, see [1], [2], [8], [9], [10], [11], [13], [14], [28] and [33].

This paper aims at proposing a method to design stabilizing lead-lag controllers for time delay systems. To the best of the authors' knowledge this problem is not fully addressed in the literature. The proposed controller is a second-order PID-like controller with three parameters to regulate. First, the complete set of all stabilizing controllers are determined. The idea is to divide the problem into two equivalent sub-problems such that only two of the controller's parameters appear in each of the sub-problems. This is achieved using Kharitonov's Lemma. This can be considered as a first step in the path of designing optimal lead-lag controllers for the studied class of time delay systems. One of the main advantages of obtaining all stabilizing lead-lag controllers is need to be tuned. Another advantage is to use to avoid stability tests each time the controller the stability regions as admissible search space for optimization techniques while avoiding stability tests in each iteration of the algorithm. For this reason, we choose to employ genetic algorithms once the complete set of stabilizing lead-lag controllers is determined, see [27] and [7].

The paper is organized as follows. Section 2 is devoted to explaining the method of determining the set of all stabilizing phase leadlag controllers for a class of time delay systems. In section 3, the genetic algorithm is introduced and then used to minimize some performance indices of the step response of the closed loop system. In Section 4, a numerical example is given to illustrate the efficiency of the proposed controller. Finally, some concluding remarks are given.

\section{Stabilizing second order controllers for time delay systems}

Given the feedback system of Fig. 1, where $r$

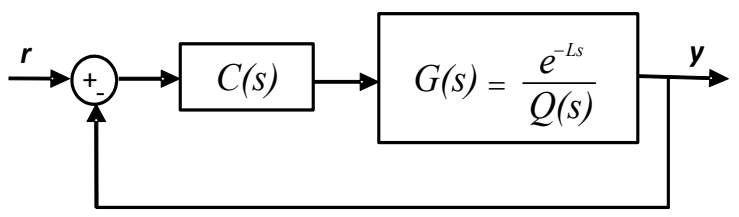

Figure 1. Classical feedback system

and $y$ are the input and the output of the system, respectively. $\mathrm{C}(\mathrm{s})$ is a second-order lead-lag controller employed to stabilize and control a 
linear time delay system whose transfer function is given by

$$
G(s)=\frac{e^{-L s}}{Q(s)}
$$

Where $\mathrm{s}$ is the Laplace operator, $Q(s)$ a polynomial in $s$, and $L>0$ represents the time delay. Many practical systems can be modelled by (1), see [24] and [33] for further details.

The lead-lag controller employed in this study is given by

$$
C(s)=\frac{\left(1+\tau_{2} s\right)\left(1+\tau_{3} s\right)}{\left(1+\tau_{1} s\right)\left(1+\tau_{4} s\right)}
$$

Where

$$
\left\{\begin{array}{l}
\tau_{1}>\tau_{2} \\
\tau_{3}>\tau_{4} \\
\tau_{2} \tau_{3}=\tau_{1} \tau_{4}
\end{array}\right.
$$

Conditions in (3) are imposed to guarantee the same gain for high and low frequencies and to combine the effects of phase lead and phase lag in certain frequency ranges and can realize the function of PID controller [5].

By straight forward modifications [4], the controller can be re-written as

$$
C(s)=\frac{s^{2}+\alpha_{3} s+\alpha_{1}}{s^{2}+\alpha_{2} s+\alpha_{1}}
$$

With this setting, the closed loop characteristic equation is given by

$$
\Delta^{*}\left(s, \alpha_{1}, \alpha_{2}, \alpha_{3}\right)=\left\{\begin{array}{l}
\left(s^{2}+\alpha_{2} s+\alpha_{1}\right) Q(s) \\
+\left(s^{2}+\alpha_{3} s+\alpha_{1}\right) e^{-L s}
\end{array}\right.
$$

In the rest of this section, we describe how to obtain the complete set of all stabilizing second order controllers for the studied class of time delay systems. The idea is to divide the problem into two equivalent sub-problems such that only two of the controller's parameters appear in each of the sub-problems. The following sub-sections describe the details of the method.

\section{A. Stability region in $\left(\alpha_{1}, \alpha_{2}\right)$ plane}

The following lemma is used to divide the original problem into two equivalent subproblems:

Kharitonov's Lemma [16]. Consider the quasi-polynomial

$$
\delta(s)=\sum_{i=0}^{n} \sum_{j=1}^{r} h_{i j} s^{n-i} e^{\tau_{j} s}
$$

such that: $\tau_{1}<\tau_{2}<\cdots<\tau_{r}$, with main term $h_{0 r} \neq 0$ and $\tau_{1}+\tau_{r}>0$ : If $\delta(s)$ is stable, then the derivative of $\delta(s)$, noted $\delta^{\prime}(s)$, is also a stable quasi-polynomial.

The idea is to reduce the number of controller's parameters in the sub-problems so that the Ddecomposition method can be used. It is straight forward to see that stability of $\Delta^{*}(s)$ given in (5) is equivalent to stability of $\Delta(s)=e^{L s} \Delta^{*}(s)$ where

$\Delta\left(s, \alpha_{1}, \alpha_{2}, \alpha_{3}\right)=\left\{\begin{array}{l}\left(s^{2}+\alpha_{2} s+\alpha_{1}\right) e^{L s} Q(s) \\ +\left(s^{2}+\alpha_{3} s+\alpha_{1}\right)\end{array}\right.$

In the rest of this paper the quasi-polynomial $\Delta(s)$ will be used to study stability of the closed loop system. Now, using Kharitonov's lemma if $\Delta(s)$ is stable then $\Delta^{\prime}(s)$ is also stable where

$\Delta^{\prime}\left(s, \alpha_{1}, \alpha_{2}, \alpha_{3}\right)=\left\{\begin{array}{l}\left(2 s+\alpha_{2}\right) P(s)+2 s+\alpha_{3} \\ +\left(s^{2}+\alpha_{2} s+\alpha_{1}\right) P^{\prime}(s)\end{array}\right.$

and

$P(s)=Q(s) e^{L s}$.

Repeating the above steps once more, if $\Delta^{\prime}(s)$ is stable then $\Delta^{\prime \prime}(s)$ is also stable, where $\Delta^{\prime \prime}(s)$ is given by

$$
\Delta^{\prime \prime}\left(s, \alpha_{1}, \alpha_{2}\right)=\left\{\begin{array}{l}
\left(s^{2} P^{\prime \prime}(s)+4 s P^{\prime}(s)\right. \\
+2 P(s)+2)+\alpha_{1} P^{\prime \prime}(s) \\
+\alpha_{2}\left(s P^{\prime \prime}(s)+2 P^{\prime}(s)\right)
\end{array}\right.
$$


Only two parameters, namely $\alpha_{1}$ and $\alpha_{2}$ appear in (10). Therefore, the Ddecomposition method, [12], [20], [21] and [29], is used to calculate the stabilizing regions in the parameter plane $\left(\alpha_{1}, \alpha_{2}\right)$.

Let

$$
\left\{\begin{array}{l}
P(j \omega)=R(\omega)+j I(\omega) \\
P^{\prime}(j \omega)=R^{\prime}(\omega)+j I^{\prime}(\omega) \\
P^{\prime \prime}(j \omega)=R^{\prime \prime}(\omega)+j I^{\prime \prime}(\omega)
\end{array}\right.
$$

Replacing s by $(j \omega)$ and equating the real and imaginary parts of (7) to zero, we get

$$
\begin{aligned}
& {\left[\begin{array}{cc}
R^{\prime \prime} & -\omega I^{\prime \prime}+2 R^{\prime} \\
I^{\prime \prime} & \omega R^{\prime \prime}+2 I^{\prime}
\end{array}\right]\left[\begin{array}{l}
\alpha_{1} \\
\alpha_{2}
\end{array}\right]} \\
& =-\left[\begin{array}{c}
-\omega^{2} R^{\prime \prime}-4 \omega I^{\prime}+2 R+2 \\
-\omega^{2} I^{\prime \prime}+4 \omega R^{\prime}+2 I
\end{array}\right]
\end{aligned}
$$

Depending on the value of $\mathrm{w}$, three cases will be investigated.

Case1: When $\omega=0$, the following equation is obtained

$$
\left.\Delta^{\prime \prime}(s)\right|_{S=0}=0
$$

or

$$
\alpha_{1}=-\frac{2 P^{\prime}(0)}{P^{\prime \prime}(0)} \alpha_{2}-\frac{2(P(0)+1)}{P^{\prime \prime}(0)}
$$

Case2: When $\omega>0$, a pair of conjugate complex roots crosses the imaginary axis. Setting the real and imaginary parts of (10) to zero, we get

$$
\left[\begin{array}{l}
\alpha_{1} \\
\alpha_{2}
\end{array}\right]=\left\{\begin{array}{c}
-\frac{1}{B_{1}}\left[\begin{array}{cc}
\omega R^{\prime \prime}+2 I^{\prime} & \omega I^{\prime \prime}-2 R^{\prime} \\
-I^{\prime \prime} & R^{\prime \prime}
\end{array}\right] \\
\times\left[\begin{array}{c}
-\omega^{2} R^{\prime \prime}-4 \omega I^{\prime}+2 R+2 \\
-\omega^{2} I^{\prime \prime}+4 \omega R^{\prime}+2 I
\end{array}\right]
\end{array}\right.
$$

where

$$
B_{1}=\left\{\begin{array}{l}
\omega R^{\prime \prime 2}(\omega)+\omega I^{\prime \prime 2}(\omega) \\
+2\left(I^{\prime}(\omega) R^{\prime \prime}(\omega)-I^{\prime \prime}(\omega) R^{\prime}(\omega)\right)
\end{array}\right.
$$

Case 3: The case of $\omega \rightarrow \infty$ represents a root leaving the left-half plane (or the right halfplane) at infinity [3], the quasi-polynomial $\Delta(s)$ possesses a root chain of retarded type.
For this reason, this case will not be considered as it does not affect the stability regions [15].

Using (13) and (14) the $\left(\alpha_{1}, \alpha_{2}\right)$ plane is divided into several regions, and stability of (10) can be tested by choosing a point inside a region and using any classical method, such as Nyquist or Bode criterion.

\section{B. Stability region in $\left(\alpha_{1}, \alpha_{3}\right)$ plane}

The second sub-problem consists of fixing one of the controller's parameters in the admissible range determined by the approach of the previous sub-section, then solving a new problem where only two parameters of the controller are unknown. We choose to fix $\alpha_{2}$ and to calculate stability regions in $\left(\alpha_{1}, \alpha_{3}\right)$ plane.

Let $P(s)=Q(s) e^{L s}$, then the characteristic function becomes

$\Delta\left(s, \alpha_{1}, \alpha_{2}, \alpha_{3}\right)=\left\{\begin{array}{l}\left(s^{2}+\alpha_{2} s+\alpha_{1}\right) P(s) \\ +\left(s^{2}+\alpha_{3} s+\alpha_{1}\right)\end{array}\right.$

Two cases are studied after replacing $\mathrm{s}$ by $(j \omega)$ and by equating the real and imaginary parts of (16) to zero.

Case1: For $\omega=0$, it comes

$$
\alpha_{1}=0
$$

Case2: For $\omega>0$, expressions of $\alpha_{1}$ and $\alpha_{3}$ are

$$
\alpha_{1}=-Y^{-1}\left\{\begin{array}{l}
-\alpha_{2}(I(\omega) \omega \cos (L \omega) \\
+R(\omega) \omega \sin (L \omega)) \\
-\omega^{2} \cos (L \omega) R(\omega) \\
+\omega^{2} \sin (L \omega) I(\omega)-\omega^{2}
\end{array}\right\}
$$

$$
\alpha_{3}=\left\{\begin{array}{l}
\omega(\sin (L \omega) R(\omega) \\
+\cos (L \omega) I(\omega)) \\
-\alpha_{2}(\cos (L \omega) R(\omega) \\
-\sin (L \omega) I(\omega)) \\
-\alpha_{1} \omega^{-1}(\cos (L \omega) I(\omega) \\
+\sin (L \omega)) R(\omega))
\end{array}\right.
$$

with

$$
Y=-I(\omega) \sin (L \omega)+R(\omega) \cos (L \omega)+1
$$


Using (17), (18) and (19) the $\left(\alpha_{1}, \alpha_{3}\right)$ plane can be divided into several regions, each region has the same number of poles in the right half of the complex plane. Stability region is determined by fixing any value of the pair $\left(\alpha_{1}, \alpha_{3}\right)$ within the region and using any classical stability method.

Repeating the above steps for the admissible values $\alpha_{2}$ allows the determination of the complete set of stabilizing lead lag controller for the system (1).

\section{Controller parameters optimiza- tion by Genetic Algorithms}

The Genetic Algorithm (GA) is an artificial optimization scheme developed in analogy to natural evolution performing an exploration of the search space [17], [18] and [19].

It has been considered as an efficient technique for searching the global or near global solution of complex optimization problems. After finding the set of all stabilizing second order controllers, a genetic algorithm method is used to find the optimal parameters values of the proposed second order controller. The principle of controller parameters optimization using the GA is given in Fig. 2, where $e, r$ and $y$ are the error, the input and the output of the closed loop system, respectively.

For the majority of the applications of genetic algorithms to solve optimization problems, the coding technique is used to represent a solution to a given problem. In this coding implementation, each chromosome is encoded as a vector of real numbers, of the same lengths as the solution vector. According to control objectives, three parameters $\alpha_{1}, \alpha_{2}$ and $\alpha_{3}$ of the studied second order controller are required to be designed. In this paper, the real-valued vector $\left[\alpha_{1}, \alpha_{2}, \alpha_{3}\right]$ is adopted as a chromosome to represent a solution to the problem; Moreover, the primary population is fixed inner the stabilizing set values of the controller, determined by the method of section II, is estimated to be 100 individuals per population and the selected generation size is 50 .

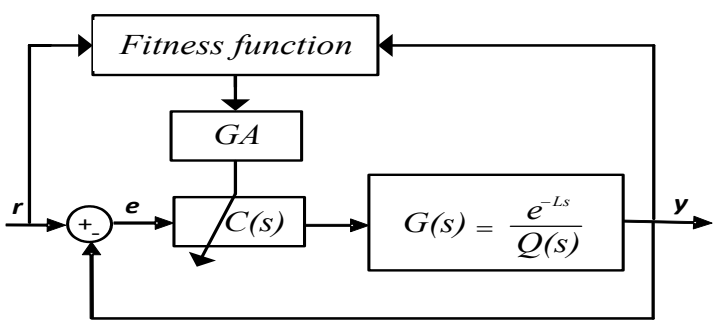

Figure 2. Principle of controller parameters optimization based GA

The use of crossover, fitness and mutation is in order to invent a new generation. Crossover, fixed as 0.8 , is a process that combines two parents and performs an interpolation along the line formed by this two parents. To transmute the generation's individuals, the mutation process is utilized. Thus, it is possible to try a completely different solution. The probability of mutation should be small in order to enable the population to improve itself by crossover; henceforth its chosen value is 0.01 . To find optimal controller, there are several performance criteria that can be evaluated, such as the Rise Time (RT), the Settling Time (ST) and the Integral Square Error (ISE) defined in [23].

$$
\begin{aligned}
& R T=t_{0.9 y_{s s}}-t_{0.1 y_{s s}} \\
& S T=\left|y(t)-y_{s s}\right| \leq 0.02 \\
& I S E=\int|e(t)|^{2} d t
\end{aligned}
$$

$y_{s s}$ is the steady state value of $\mathrm{y}(\mathrm{t}), t_{\gamma y_{s s}}$ the time to reach $\gamma \%$ of $y_{s s}$ and $e(t)$ the error signal in time domain.

The steps of calculating parameters using GA, are as follows [7] and [30]

Step 1: Generate a fixed-size initial random population of individuals.

Step2: Calculate the fitness for each string in the population.

Step3: Evaluate the initial population.

Step4: Create offspring using genetic operators and invent a new population.

Step5: Growth of the recent reproduced population.

Step6: Stop, if the search goal is achieved. Otherwise, repeat with Step4. 
This GA will be used in the next section to optimize section II results.

\section{Example}

In this example, the proposed method is applied on a stable third-order system

$$
G(s)=\frac{e^{-0.2 s}}{2 s^{3}+2.5 s^{2}+3 s+1}
$$

whose open-loop step response is given in Figure 3 . The first step in the design procedure is to determine the set of stabilizing regions.

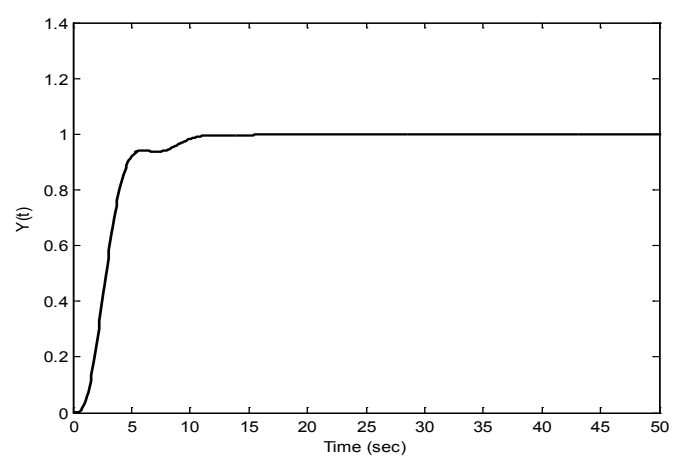

Figure 3. Step response of the studied open loop system

The admissible stabilizing values of $\left(\alpha_{1}, \alpha_{2}\right)$. are calculated using (13) and (14), which are in turn derived using characteristic function given by

$$
\begin{aligned}
\Delta^{\prime \prime}\left(s, \alpha_{1}, \alpha_{2}\right)= & \left\{\begin{array}{l}
0.08 s^{5}+\left(4.1+0.08 \alpha_{2}\right) s^{4} \\
+\left(44.12+3.3 \alpha_{2}+0.08 \alpha_{1}\right) s^{3} \\
+\left(33.64+27.12 \alpha_{2}+2.5 \alpha_{1}\right) s^{2} \\
+\left(18.8+17.44 \alpha_{2}+14.12 \alpha_{1}\right) s \\
+6.4 \alpha_{2}+6.24 \alpha_{1}
\end{array}\right\} e^{0.2 s} \\
& +2
\end{aligned}
$$

The stability region for this sub-problem is shown in Fig. 4.

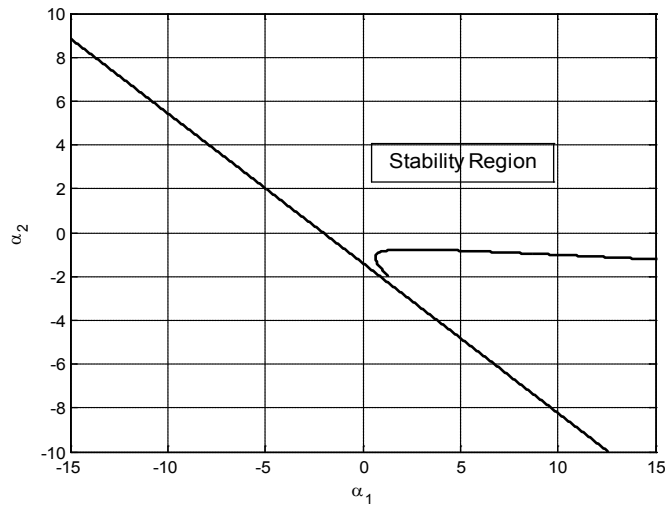

Figure 4. Stability region in the $\left(\alpha_{1}, \alpha_{2}\right)$ plane

To show the second part of the method, let us choose a value of $\alpha_{2}$ from this stability region, for example $\alpha_{2}=1.5$, and apply (17), (18) and (19), to obtain the stability region in the $\left(\alpha_{1}, \alpha_{3}\right)$ plane, given in Fig. 5. Fig. 6 shows a 3$\mathrm{D}$ plot of the stability regions for, $\alpha_{2} \in\left[\begin{array}{ll}1.5 & 2\end{array}\right]$.

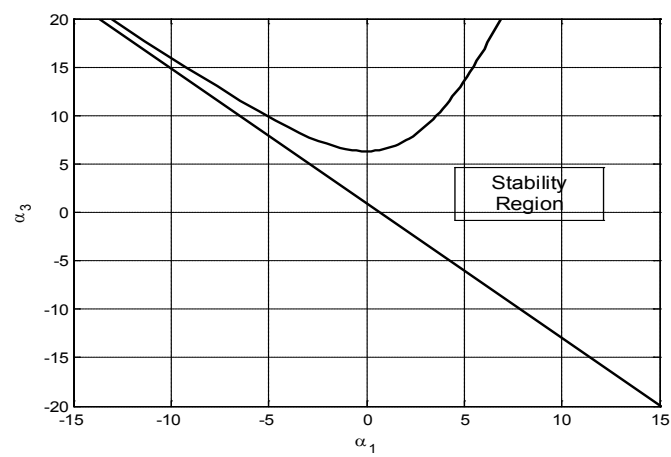

Figure 5. Stability region in the $\left(\alpha_{1}, \alpha_{3}\right)$ plane obtained for $\alpha_{2}=1.5$

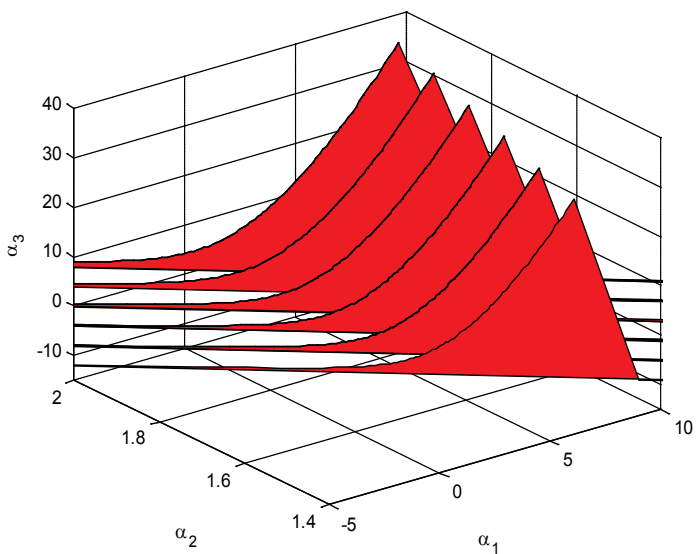

Figure 6. Stability regions obtained for $\alpha_{2} \in\left[\begin{array}{ll}1.5 & 2\end{array}\right]$ 
Let's consider choosing $\alpha_{1}$ and $\alpha_{3}$ such that: $\alpha_{1}=1$ and $\alpha_{3}=3$. The lead-lag parameters values then, in the form (3), are determined and they are: $\tau_{1}=0.51, \tau_{2}=0.39, \tau_{3}=2.61$ and $\tau_{4}=0.99$. It allows us to deduce that conditions (4) are verified.

Once the stabilizing values of $\alpha_{1}, \alpha_{2}$ and $\alpha_{3}$ are obtained, the genetic algorithm is applied to minimize the ISE, RT and ST performance indices. The upper and lower bounds of the parameters $\left(\alpha_{1}, \alpha_{2}, \alpha_{3}\right)$ are within the following ranges: $\left[\begin{array}{ll}0 & 15\end{array}\right],\left[\begin{array}{ll}0 & 10\end{array}\right]$ and [-20 20], respectively.

In Table 1, a comparison is made between the performances of the second order controller obtained using GA and the second order controller without optimization.

Table 1. Performances of the obtained second order controllers

\begin{tabular}{|l|c|c|c|c|}
\hline & $\left(\alpha_{1}, \alpha_{2}, \alpha_{3}\right)$ & ISE & RT & ST \\
\hline $\begin{array}{l}\text { Case1: Second } \\
\text { order controller }\end{array}$ & $(1.50,3.00,0.50)$ & 1.62 & 6.10 & 12.80 \\
\hline $\begin{array}{l}\text { Case2: Second } \\
\text { order controller } \\
\text { based GA }\end{array}$ & $(3.97,7.73,6.41)$ & 1.55 & 3.50 & 12.60 \\
\hline
\end{tabular}

In case $1, \alpha_{1}, \alpha_{2}$ and $\alpha_{3}$ values are chosen arbitrarily within the upper and lower bounds, and in case 2 the controller's parameters are determined by applying the GA. As can be seen in Table 1 and Fig. 7, minimizing the ISE leads to an oscillatory response and an important peak with a short Rise Time and Settling Time.

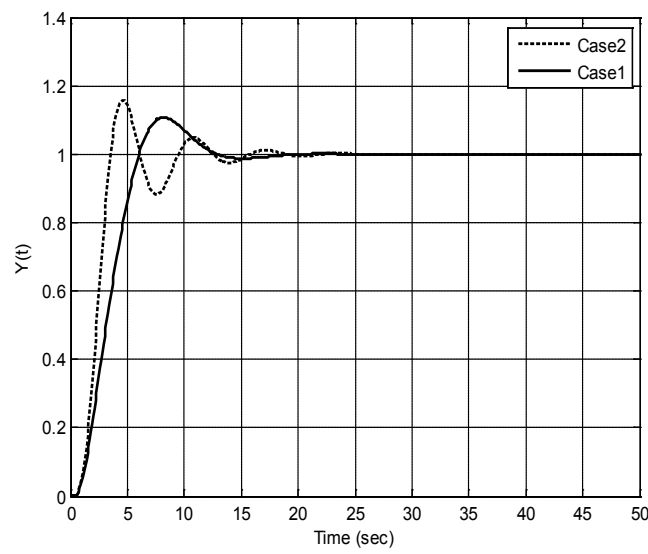

Figure 7. Step responses of the closed loop system without (case1) and with (case2) using GA technique

\section{Conclusion}

In this paper, a lead-lag controller is used to stabilize a class of linear time delay systems. The proposed approach is based on using the D-decomposition method to determine, first, the stability range of the controller's parameters $\alpha_{1}$ and $\alpha_{2}$, then one of these parameters is fixed within the admissible range and the stability regions in the space of the remaining two parameters are determined. Once the stabilizing regions are found, some performance can be evaluated such that the integral square error, the rise time and the settling time and then an optimal second order controller could be obtained using the genetic algorithm optimization method.

\section{REFERENCES}

1. Alikhani H. \& Madady A. (2013). Firstorder controller design for second order integrating systems with time delay. in Proceedings of IEEE Conference on Control Applications, Hyderabad.

2. Amri I., Soudani D.,Benrejeb M. (2010). Delay dependent robust expo-nential stability criterion for perturbed and uncertain neutral systems with time varying delays. Studies in Informatics and Control, 19 (2), pp.135-144. 
3. Bellman R.E.,Cooke K.L. (1963). Differential-difference equations. Academic Press, New York.

4. Ben Hassen N., Saadaoui K., Benrejeb M. (2015). Stabilizing time delay systems with prespecified gain and phase margins by lead-lag controllers. Int. Journal of Systems Applications Engineering \& Development, 9, pp. 47-53.

5. Borne P., Dauphin-Tanguy G., Richard J.P., Rotella F. \& Zambettakis I. (1993). Analyse et régulation des processus industriels. Tome I: Régulation continue. Editions Technip, Paris.

6. Chen C.K., Kuo H.H., Yan J.J., Liao T.L. (2009). GA-based PID active queue management control design for a class of TCP communication networks. Expert Systems with Application on Science Direct, 36 (2), pp. 1903-1913

7. Chen H.C.,Chang S.H. (2006). Genetic Algorithms based optimization design of a PID controller for an active magnetic bearing. Int. Journal of Computer Science and Network Security, 6, pp. 95-99.

8. Dang Q.V., Dequidt A., Vermei-Ren L., DAMBRINE M. (2014). Design and control of force feedback haptic systems with time delay. Springer Int. Publishing, pp.373-387.

9. Elmadssia S., Saadaoui K., M., Benrejeb (2012). PI controller design for time delay systems using an extension of the HermitBiehler theorem. Journal of Industrial Engineering, Vol. 13.

10. Elmadssia S., Saadaoui K., Benrejeb M. (2016). New stability conditions for nonlinear time varying delay systems. Int. Journal of Systems Science, 47, pp. 2009-2021.

11. Efimov D., Polyakov A., Perru-Quetti W., Richard J.P. (2016). Weighted homogeneity for time delay systems: Finite time and independent of delay stability. IEEE Trans. on Automat. Cont., 61, pp. 210-215.
12. Gryazina E.N., Polyak B.T. (2006). Stability regions in the parameter space: Ddecomposition revisited. Automa-tica, 42, pp. 13-26.

13. Guerra R.G.V., Rubio J.F.M., Cuéllar B.M. \& Sánchez G.I.D. (2016). Dynamic delayed controllers for unstable recycling systems with time delays. Studies in Informatics and Control, 25(2), pp. 195-206.

14. Hetel L., Fiter C., Omran H., Seuret A., Fridman E., Richard J.P. \& Niculescu S.I. (2017). Recent developments on the stability of systems with aperiodic sampling: an overview. Automatica, 76, pp. 309-335.

15. Hohenbicher N., Acker-Mann, J. (2003). Computing stable regions in parameter spaces for a class of quasi-polynomials. in Proceedings of 4th IFAC Workshop on Time Delay Systems, TDS' 03, Rocquencourt.

16. Kharitonov V.L., Niculescu S., Moreno J., MICHIELS W. (2005). Static out-put stabilization: Necessary conditions for multiple delay controllers. IEEE Trans. on Automat. Cont., 50, pp.82-86.

17. Marra M.A., Walcott B.L. (1996). Stability and optimality in genetic algo-rithm controllers. in Proceedings of IEEE International Symposium on Intelligent Control, Dearborn.

18. Masum A.K.M., Shahjalal M., Faruque M.F., Sarker M.I.H. (2011). Solving the vehicle routing problem using genetic algorithm. Int. Journal of Advanced Computer Science and Applications (IJACSA), 2(7), pp. 126-131.

19. Ohri J., Kumar N. \& Chinda M. (2014). An improved genetic algorithm for PID parameter tuning. Recent Advances in Electrical and Computer Engineering, pp. 191-198, Venice.

20. Osusky J. \& Vesely V. (2010). Modification of Neimark D-partition method for desired phase margin. in Proceedings of the International Conference on Cybernetics and Informatics, Vyšná Boca.

21. Pandey I.K. \& Dewan L. (2014). Stabilizing sets of PID controllers for minimum phase integrating processes with dead 
time. in Proceedings of the 13th Int. Conference on Circuits, Systems, Electronics, Control \& Signal Processing, CSECS, Lisbon.

22. Pekar L. \& R. Prokop (2010). Non-delay parameter depending stability of a time delay system. Proceedings of 14th WSEAS Int. Conf. on Systems, Corfu.

23. Pillai R.P., Jadhav S.P. \& Patil M.D. (2013). Tuning of PID controllers using advanced genetic algorithm. Int. Journal of Advanced Computer Science and Applications (IJACSA), Special issue of selected paper, pp. 1-6.

24. Rico J.E.N. \& Camacho E.F. (2007). Control of dead time processes. London, Springer.

25. Saadaoui K. (2003). Fixed order controller design via parametric methods. Ph. D Thesis, Bilkent University.

26. Saadaoui K. \& Ozguler A.B. (2009). Stabilizing first-order controllers with desired stability region. Control and Intelligent Systems, 37, pp. 31-38.

27. Saadaoui K., Moussa A., \& Benrejeb, M. (2009). PID controller design for time delay systems using heneric algorithms. The Mediteranean Journal of Mwasurement and Control, 5, pp. 31-36.
28. Saadaoui K., Testouri S. \& Benrejeb M. (2010). Robust stabilizing first order controllers for a class of time delay systems. ISA Transactions, 49, pp. 277-282.

29. Saadaoui K., Ben Hassen N., Benrejeb M. (2015). Stabilizing time delay systems by PID controllers. 2nd Int. Conference on Automation, Control, Engineering and Computer Science, Proceedings of Engineering \& Technology, ACECS, Sousse.

30. Serban C., Carp D. (2016). Optimization of container stowage in a yard block using a genetic algorithm. Studies in Informatics and Control, 25 (1), pp. 123-130.

31. Tan N., Kaya I., Yeroglu C., Atherton D.P. (2006). Computation of stabilizing PI and PID controllers using the stabilizing boundary locus. Energy Conver-sion \& Management, 47, pp. 3045-3058.

32. Wang H., Vasseur C., Koncar V., Chamroo A. \& Christov N. (2010). Sampled tracking for delayed systems using two-time-scale sampled data controllers. Studies in Informatics and Control, 19 (4), pp. 339-346.

33. Zhong Q.C. (2006). Robust control of time delay systems. London, Springer. 